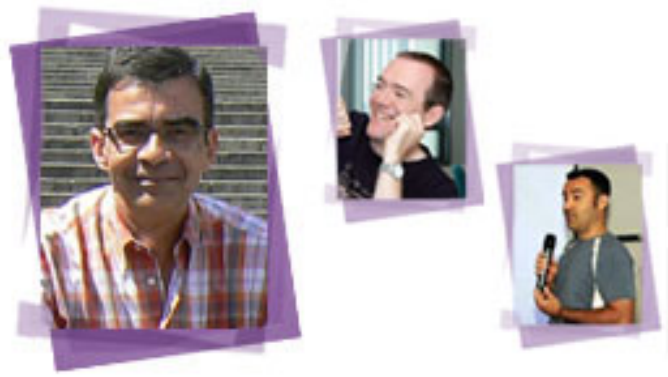

\section{Traducir EUROPA: un caso práctico de gestión multilingüe de un sitio web}

Carlos Nogueira (con la colaboración de David Alford y Pedro Blanco)

Unidad de Traducción Web

Dirección General de Traducción, Comisión Europea

\title{
Resumen
}

Este artículo se refiere a la importancia del multilingüismo en las instituciones de la UE y a la política de comunicación europea a través de Internet y describe el funcionamiento de la unidad de traducción Web, específicamente creada en la Comisión Europea para editar/traducir los textos destinados al portal Europa. Incluye una parte sobre el trabajo de edición de los textos originales y otra dedicada al punto de vista del traductor.

\section{Palabras clave}

multilingüismo, localización, Internet, Europa, Comisión Europea, edición, traducción

\section{El multilingüismo en la UE}

Desde sus primeros tiempos, la Unión Europea viene demostrando la importancia que otorga a las lenguas. El Reglamento $\mathrm{n}^{\circ} 1$ de la Comunidad Económica Europea (Reglamento, 1958), adoptado en 1958 y que sigue en vigor (con las necesarias modificaciones tras cada adhesión), establece el régimen lingüístico de las instituciones europeas.

Ese mismo reglamento determina las lenguas oficiales y de trabajo de las instituciones y deja a cada institución la libertad de determinar las modalidades de aplicación del régimen lingüístico en sus reglamentos internos. Por otro lado, con las sucesivas ampliaciones y la preocupación más reciente de comunicar mejor con el ciudadano, el multilingüismo se ha convertido en una de las políticas de la Unión, integrada en la cartera de uno de los Comisarios.

\section{La traducción}

La importancia del multilingüismo en el marco de una organización internacional como la UE se traduce, en la práctica, en la necesidad permanente de interpretar (reuniones de trabajo, cumbres de jefes de gobierno, etc.) y de traducir (actos legislativos, documentos de trabajo, actas de reuniones, etc.).

Cada institución de la UE (Parlamento, Consejo, Comisión, etc.) cuenta con un servicio de traducción, organizado según sus necesidades.

El servicio de traducción de la Comisión Europea (Dirección General de Traducción o DGT) es uno de los mayores del mundo (1512 traductores, traducción hacia 23 lenguas, 506 combinaciones posibles, ...).

Traducir EUROPA: un caso práctico de gestión multilingüe de un sitio web. Carlos Nogueira (c/colab. de David Alford y Pedro Blanco). Unidad de Traducción Web D G T. Comisión Europea Revista Tradumàtica - Traducció i Tecnologies de la Informació i la Comunicació 
Está organizado por departamentos lingüísticos (uno por cada lengua), y dentro de cada departamento, por unidades temáticas, lo que permite un cierto grado de especialización a los traductores.

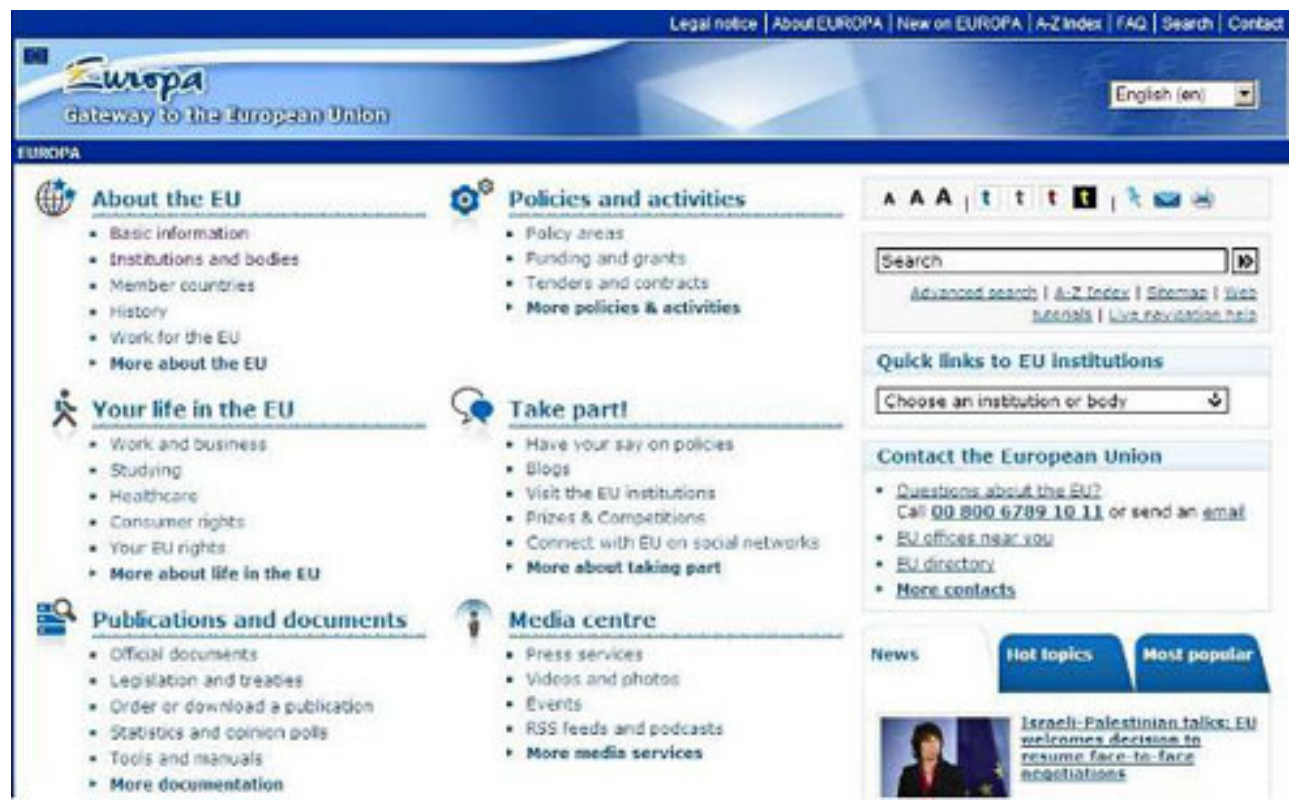

\section{El portal Europa}

En los últimos años ( $y$, sobre todo, tras la votación negativa registrada en 2005 en Francia y en los Países Bajos sobre la Constitución), la Unión Europea ha buscado mejorar la comunicación con los ciudadanos, al mismo tiempo que reconocía que Internet se ha convertido no solo en un foro de debate político de primer orden, sino también en un canal de comunicación y una fuente de información privilegiados.

En uno de los muchos documentos de reflexión publicados en la época por la Comisión sobre el tema, el Libro Blanco sobre una política europea de comunicación (Libro Blanco, 2006) se afirma que todos los ciudadanos deberían tener acceso en su propia lengua a información sobre los asuntos públicos y que la información debe difundirse ampliamente a través de una gran variedad de canales, incluidos los medios de comunicación y las nuevas tecnologías, como Internet.

En 1995 fue lanzado el portal Europa (http://europa.eu/index en.htm), que es el punto de acceso único a todos los sitios y páginas Web de todas las instituciones y organismos de la UE. Considerado uno de los mayores portales del mundo, su gestión es responsabilidad de la Comisión Europea (un Comité Editorial Interinstitucional asegura la coherencia entre los varios sitios Web de Europa). 
Su objetivo es informar a los ciudadanos en su propia lengua. Sin embargo, el número de lenguas disponibles en las páginas de Europa varía según el sitio y, en un mismo sitio, según los varios niveles de información.

La legislación y los documentos de gran importancia política se publican en las 23 lenguas oficiales de la UE. La información de actualidad o destinada a un público especializado no siempre aparece en todas las lenguas. Además del inglés (o inglés, francés y alemán), la elección de las lenguas en las que se publican las páginas de Europa se hace en función de las lenguas más utilizadas o conocidas del público al que se destinan.

\section{Nueva unidad de traducción}

La necesidad de una unidad específicamente reservada a la Web se ha hecho más apremiante con el desarrollo exponencial del sitio Web Europa, dado que traducir para la Web exige cualificaciones especiales, tanto lingüísticas como técnicas.

La unidad de traducción Web de la DGT fue oficialmente creada en marzo de 2006, con el objetivo de traducir las páginas Web de la Comisión Europea.

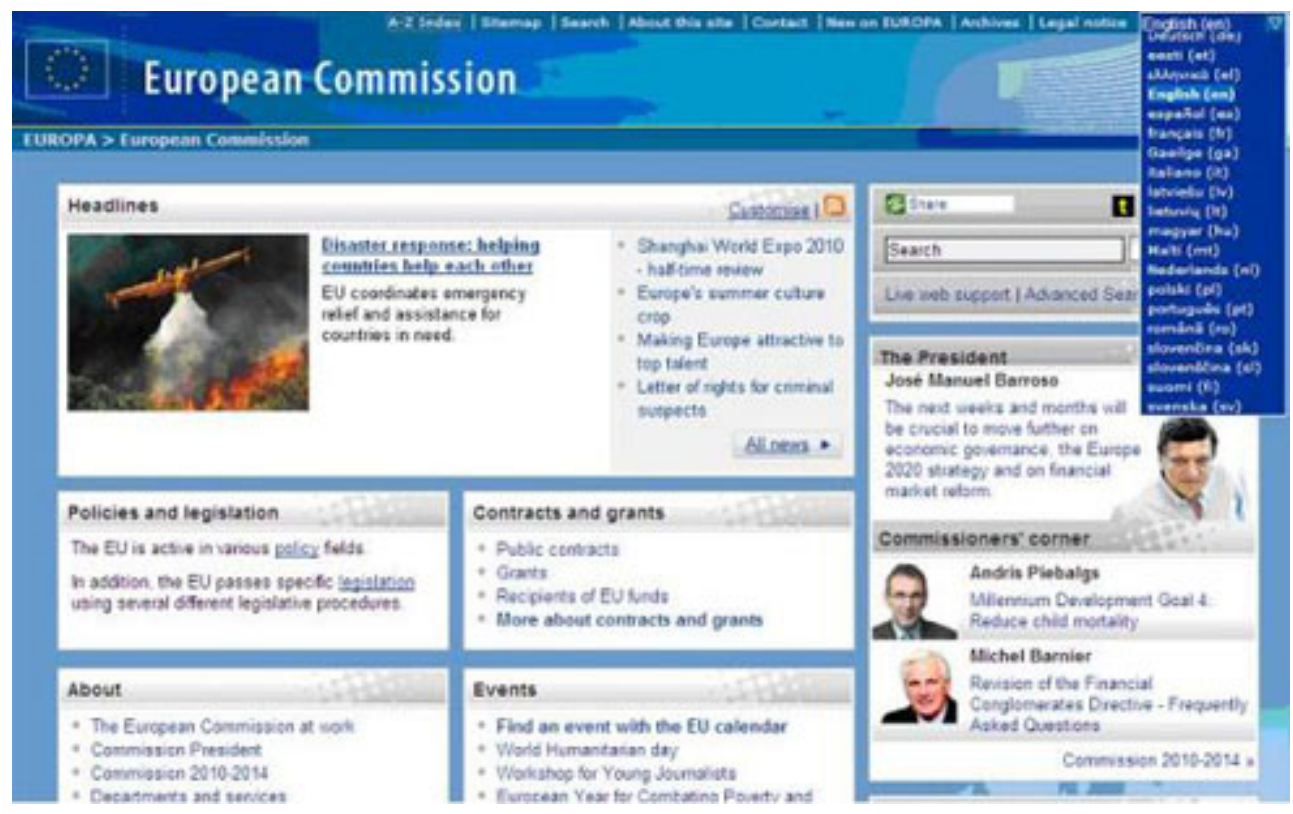

\subsection{Características distintivas}

Cada lengua oficial está representada por 4 a 6 traductores (con excepción del irlandés, que cuenta con 1 traductor) y la unidad cuenta también con 6 asistentes. Para facilitar la gestión de una unidad gigante (casi tan grande como un departamento lingüístico), los distintos equipos lingüísticos fueron agrupados

Traducir EUROPA: un caso práctico de gestión multilingüe de un sitio web. Carlos Nogueira (c/colab. de David Alford y Pedro Blanco). Unidad de Traducción Web D G T. Comisión Europea Revista Tradumàtica - Traducció i Tecnologies de la Informació i la Comunicació

08: Localització i web : ISSN: 1578-7559

http://wnww.fti.uab.cat/tradumatica/revista 
en tres sectores (cada uno bajo un jefe de sector). La unidad tiene también su propio planning, cuyo jefe es responsable por la gestión de la demanda.

\begin{tabular}{|l|}
\hline UNIDAD DE TRADUCCIÓN WEB EN NÚMEROS \\
(Septiembre 2010)
\end{tabular}

La organización del trabajo en la unidad es muy distinta de las otras unidades en los departamentos lingüísticos "tradicionales". Mientras las otras unidades de traducción de la Dirección General de Traducción se componen de traductores (y asistentes) de la misma lengua materna que sólo traducen hacia esa lengua, la principal característica de la unidad de traducción Web es que traduce para todas las lenguas oficiales; por lo tanto, incluye traductores de todas las lenguas oficiales de la UE, lo que da un colorido muy especial a los contactos entre los profesionales que la integran. En la práctica, su babelismo ha sido resuelto con la adopción del inglés como lingua franca, utilizado en las reuniones de trabajo y en la comunicación oral y escrita de sus miembros.

Dentro de cada uno de los 23 equipos lingüísticos que forman la unidad, los traductores generalmente se turnan en la tarea de atribución de la traducción y revisión a través de la aplicación Suivi. Todos los textos son revisados por colegas del equipo, sin que haya una separación de funciones entre traductores y revisores.

En general, los plazos en la unidad de traducción Web son cortos. Los textos son también relativamente cortos en comparación con los traducidos por los departamentos lingüísticos. Eso se debe a que es más difícil leer en una pantalla que en papel. La regla "KISS" (Keep It Short and Simple) seguida por los periodistas en general es ineludible al escribir - y traducir - para la Web.

\begin{tabular}{|c|c|c|}
\hline \multicolumn{3}{|c|}{$\begin{array}{l}\text { Principales diferencias entre la unidad de traducción Web y las unidades de traducción de los } \\
\text { departamentos lingüísticos }\end{array}$} \\
\hline & Traducción Web & $\begin{array}{l}\text { Unidades de traducción de los } \\
\text { departamentos lingüísticos }\end{array}$ \\
\hline $\begin{array}{l}\text { Tamaño de los documentos } \\
\text { (promedio de páginas) }\end{array}$ & 2,4 & 5,7 (media de la DGT) \\
\hline Plazos de 2 días o menos & $29,1 \%$ & 10,8\% (media de la DGT) \\
\hline Formatos del texto original & $\begin{array}{c}\text { DOC (con hipervínculos), } \\
\text { HTML, XML, txt, sitios Web } \\
\text { completos en archivos ZIP, } \\
\text { PPT, aplicaciones en linea, XLS }\end{array}$ & DOC, XLS \\
\hline Herramientas & $\begin{array}{c}\text { TWB, TagEditor, diferentes } \\
\text { CMS }\end{array}$ & TWB \\
\hline
\end{tabular}

Fuente: Unidad DGT.S.4 


\section{>> revista tradumàtica}

Traducció i Tecnologies de la Informació i la Comunicació

Número 08: Localització i web : desembre 2010 :

http://www.fti.uab.cat/tradumatica/revista : ISSN: 1578-7559

La unidad es responsable por la traducción de sitios Web completos (por ejemplo, el portal Salud-UE, http://ec.europa.eu/health-eu/index es.htm), así como nuevas páginas Web y actualizaciones de páginas existentes (contenidos o frecuentemente sólo los menús).

Una de sus tareas estándar es la traducción de los artículos de información diarios publicados en todas las lenguas oficiales de la UE en la página principal de la Comisión Europea

(http://ec.europa.eu/index es.htm). El original (en inglés o francés) de los artículos llega para edición por los traductores ingleses o franceses pronto por la mañana y, después, el texto editado, que debe ser aprobado por la DG Comunicación, se convierte en el texto original para la traducción hacia todos los otros idiomas. Por lo general, las traducciones deben estar listas para las cuatro de la tarde. A pesar de que estos plazos breves obligan a los traductores a trabajar rápidamente, el trabajo es gratificante porque en pocas horas se puede leer en Internet el texto que se acaba de traducir.

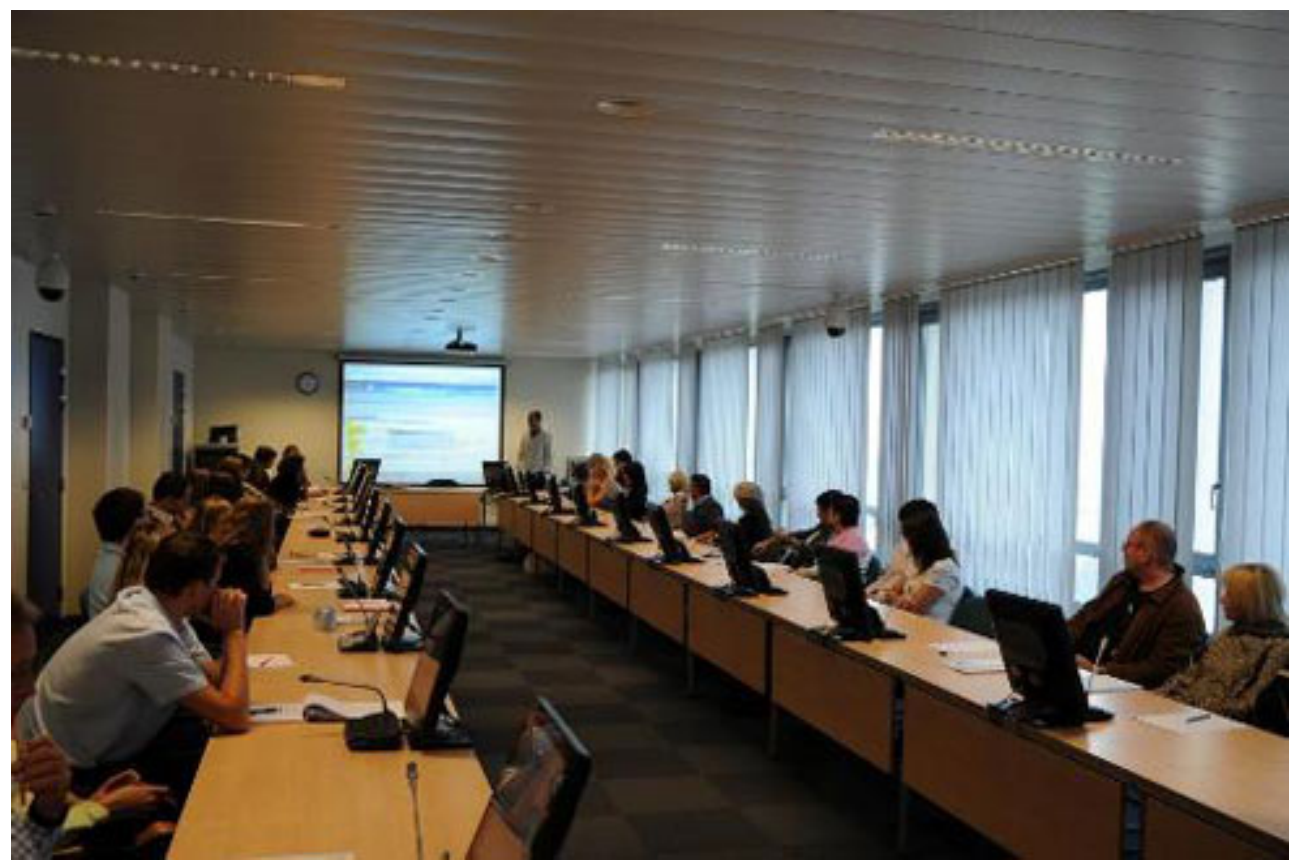

Pie de foto: Presentación de una nueva herramienta en una reunión de unidad

\subsection{Herramientas específicas}

Los traductores Web trabajan en una gran variedad de formatos de texto, que requieren herramientas diversas. Para traducir documentos en HTML o en XML, utilizan TagEditor junto con Translator's Workbench (TWB), un programa de memorias de traducción. Esto permite que los traductores traduzcan el texto sin tocar las etiquetas, que vean cuál será el aspecto del texto en la pantalla después de la publicación en la Web y que adapten los titulares según el espacio disponible. Además, hacen traducciones y correcciones directamente en el WCMS central de la Comisión y utilizan diferentes interfaces de traducción en línea.

Traducir EUROPA: un caso práctico de gestión multilingüe de un sitio web. Carlos Nogueira (c/colab. de David Alford y Pedro Blanco). Unidad de Traducción Web D G T. Comisión Europea Revista Tradumàtica - Traducció i Tecnologies de la Informació i la comunicació

08: Localització i web : ISSN: 1578-7559

http://wnw.fti.uab.cat/tradumatica/revista 


\subsection{Edición de los originales}

Dado que la gran mayoría de los originales son en inglés, los cinco traductores ingleses de la unidad Web desempeñan un papel decisivo en la edición, mejorando y abreviando los textos y adaptándolos a la Web.

\subsection{Lenguaje de todos los días y localización}

A diferencia de lo que ocurre en los departamentos "tradicionales" de la DGT, la traducción para la Web no depende de una terminología técnica o jurídica especializada. Los traductores Web evitan la jerga propia de las instituciones europeas y adoptan un estilo periodístico accesible al ciudadano medio, dado que los textos, por lo menos en los primeros niveles de información, se destinan esencialmente a un público no especializado.

Además, hay que tener en cuenta que, según algunos estudios, la lectura en una pantalla es un $25 \%$ más difícil que en papel y que es sabido que los internautas tienden a "saltarse" rápidamente el texto clicando en un enlace que les permita seguir navegando.

Siempre que es posible, se intenta "localizar" el texto, como forma de acercarlo al lector. No sólo se busca que el texto no parezca una traducción, sino que, en ocasiones, se adaptan los contenidos para que el lector, en su país, conecte más fácilmente con el texto.

\section{Referencias}

Libro Blanco (2006) - Libro Blanco sobre una política europea de comunicación (Comisión Europea, 1 febrero 2006):

http://eur-lex.europa.eu/LexUriServ/LexUriServ.do?uri=COM:2006:0035:FIN:ES:PDF

Reglamento (1958) - Reglamento $n^{\circ} 1$ de la Comunidad Económica Europea (Comunidad Económica Europea, 1958):

http://eur-lex.europa.eu/LexUriServ/LexUriServ.do?uri=CELEX:31958R0001:ES:HTML 


\section{APÉNDICE 1}

\section{Helping Mr Schmitt: DGT hits the road to spread the word about good web writing}

Dave Alford

DGT's web unit has a mission to make life easier for the ordinary European who might stumble onto the Commission's web pages - let's call him Citizen Schmitt.

Like most people, when Mr Schmitt logs on to the internet after a hard day's work, he's looking for something specific. He doesn't want to while away his evening reading long tracts on the "three Communities" or the cryptically-titled Lisbon Strategy.

But he might be looking for information on how he can work in another European country, whether his daughter can get a grant to study abroad or even how to get funding for a local environmental project. The key thing is, he wants that information fast, because his dinner's getting cold, he still has to fix that dripping tap and the big match starts in an hour...

If the Commission wants to communicate with Citizen Schmitt through the internet, we need to realise that it's not enough simply to post up "information" - it has to be the right information, and the right amount. And above all it needs to be presented in language the man in the street will not only recognise, but is also likely to use when googling - so not "SMEs", please, but "small businesses". And no "third countries" (what happened to countries one and two?) but "non-EU countries", or even just "other countries" or "partner countries" - whatever fits the context.

Mostly this is a question of avoiding the jargon we in the Brussels bubble all know and love - but which, to the rest of the world, sounds like Chinese. But it's also about putting ourselves in the shoes of the average person, trying to think like they do.

This is where DGT's web unit can help, as it provides services that go far beyond translating. As a first step, we give feedback to improve the usability of translated web pages. But where authoring DGs are prepared to work with us at an early stage of drafting, we can also offer native-speaker linguistic advice and editing of original texts, to make sure the basis for translation is sound.

By ironing out terminology/clarity/style problems before translation, we can produce quicker and better translations, avoiding the multiplication of errors and over-long text in up to 22 language versions.

Because words are the key content on a webpage, using the right ones in every language should mean that people can find our sites through search engines and navigate around them easily, not to mention projecting a professional image for the Commission, based on quality not quantity.

It's not enough simply to post up"information" - it has to be the right information, and the right amount. 
Slowly, this message is getting through, and some authoring units have already started working closely with us. In the long run, this should make life easier for everyone, including the long-suffering Mr Schmitt!

Use your reader's words - examples of how we can think and write from the reader's perspective.

Because words are the key content on a web page, using the right ones in every language should mean that people can find our sites through search engines and navigate around them easily.

\begin{tabular}{|l|l|}
\hline ORIGINAL TEXT & SUGGESTED ALTERNATIVE \\
\hline $\begin{array}{l}\text { Free circulation of workers } \\
\text { moving around Europe to live, work and study. } \\
\begin{array}{l}\text { Formulated like this, it merely reflects an abstract, } \\
\text { top-down economist's perspective... }\end{array}\end{array}$ & $\begin{array}{l}\text { Working abroad } \\
\text { Much more likely to attract a click from e.g. a } \\
\text { formalities involved in working abroad. }\end{array}$ \\
\hline $\begin{array}{l}\text { SME } \\
\begin{array}{l}\text { A much-loved Brussels staple, but you won't find } \\
\text { any budding entrepreneurs in the UK asking their } \\
\text { bank manager for money to start an SME... }\end{array}\end{array}$ & $\begin{array}{l}\text { Small and medium-sized enterprise is specialist } \\
\text { economic terminology in English. As the acronym } \\
\text { SME it is even rarer. } \\
\text { Small business effectively implies "medium-sized" } \\
\text { too, i.e. anything that's not big. }\end{array}$ \\
\hline $\begin{array}{l}\text { Expeditiously } \\
\text { Legal language - did you do your work } \\
\text { expeditiously today? }\end{array}$ & $\begin{array}{l}\text { Well } \\
\text { No, you (hopefully) did it well }\end{array}$ \\
\hline $\begin{array}{l}\text { Member State } \\
\text { When was the last time someone you met in a } \\
\text { bar asked you which Member State you were } \\
\text { from? This is diplomatic language. }\end{array}$ & $\begin{array}{l}\text { (EU) country } \\
\text { Yes, most of our friends and family probably live }\end{array}$ \\
\hline $\begin{array}{l}\text { Enterprise } \\
\text { False friend from French. In English, more likely } \\
\text { to bring to mind Star Trek }\end{array}$ & $\begin{array}{l}\text { Business } \\
\text { That's more like it. }\end{array}$ \\
\hline
\end{tabular}


Useful information on specific provisions contained in Belgian law

Top heading on a page, with minimal other indications of context

Nice to know that the information is useful and the provisions specific - but what is it about?!

\section{Belgian rules on package travel}

Much shorter alternative which removes the empty words and puts in the actual content.

\section{APÉNDICE 2}

\section{Charla con un traductor español de la Web, Pedro Blanco}

¿Qué te animó a dejar el trabajo de traductor tradicional para unirte a la unidad web? Bueno, la unidad web abría una puerta a un tipo de comunicación inmediata, una posibilidad de contarle directamente a la gente cosas que les afectan en la vida cotidiana. Ese aspecto novedoso me atrajo desde el primer momento.

\section{¿En qué se diferencia la traducción web de la traducción tradicional?}

Ambas persiguen objetivos distintos. En la traducción institucional clásica la fidelidad al original es el "primer mandamiento", ya que en muchos casos se trata de textos jurídicos, informes o cartas que deben surtir el mismo efecto en varios idiomas. En la traducción web la atención no se centra tanto en la letra del mensaje, sino en su espíritu. Lo fundamental es facilitarle la vida al lector.

\section{¿Podrías darnos algún ejemplo?}

En una Directiva la responsabilidad del traductor por las dificultades que pueda plantear la comprensión del texto es limitada. Aunque siempre debe buscar la redacción más clara posible, en el fondo sabe que se trata de un texto intrínsecamente difícil que exigirá un esfuerzo por parte del lector. En cambio, cuando navegamos por la red todos nos convertimos en consumidores ávidos de información, dispuestos a pasar a otra página en unos pocos segundos si no encontramos nada que nos parezca útil. Nadie va a la web para releer varias veces un párrafo difícil de entender.

\section{¿Cómo se consigue atrapar la atención del internauta?}

La presentación visual de los contenidos es un aspecto fundamental, en la que trabajan principalmente nuestros compañeros del equipo de edición. La contribución de los traductores es esencialmente lingüística y consiste en dar con la formulación más accesible para la idea que se quiere transmitir. En los primeros tiempos de la unidad, para dar con el nivel de registro adecuado me imaginaba una conversación con un familiar o un amigo. Cuando se pone cara al destinatario todo se hace más sencillo. La localización del mensaje también facilita la comprensión. 


\section{¿Qué es la localización del mensaje?}

A menudo se trata de un pequeño cambio respecto al texto original que sirve para situar el mensaje en un marco de referencia más familiar para el lector. Por ejemplo, en los textos de la Comisión Europea se utiliza frecuentemente la sigla CET (Central European Time, es decir, hora de Europa Central) para señalar la hora a la que expira un plazo o se realiza una actividad. En las traducciones más técnicas suele conservarse esa referencia. Sin embargo, en la traducción web solemos cambiarla por el equivalente "hora peninsular española", más familiar para nuestros lectores.

\begin{tabular}{l} 
European language fest \\
Culture education and youth $-24 / 09 / 2009$ \\
\hline
\end{tabular}

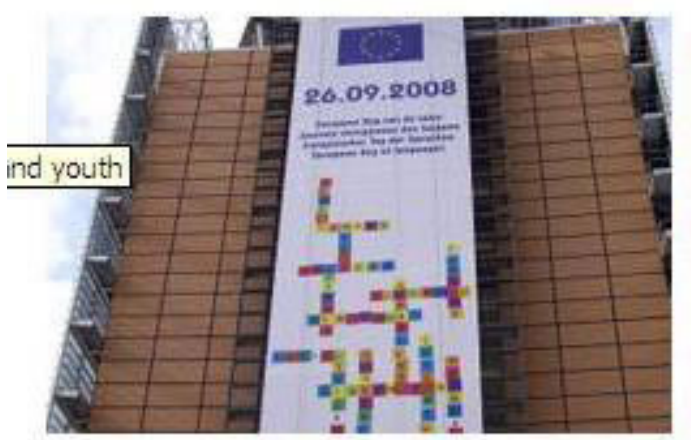

European day of languages is a celebration of the many languages spoken in the EU. Check out the events taking place in your country in the week leading up to 26 September.

The idea is to promote interest in languages and language learning for adults and children So whatever your age and whatever your interest in language, there should be something for you among the $300+$ events (over 50 in the UK and Ireland alone):

Activities range from the light-hearted to the serious - from games and quizzes to literary evenings and round-table discussions on language policy.

If you're in central London on 25 September, drop in on the language stand at Victoria station and enter a competition to win an Italian course. Or find out more about language courses for adults at events dotted around the UK.

In Brussels and Luxembourg - the EU commission's main sites - events will include a conference and press event on early language learning with multilingualism commissioner Leonard Orban (24-25 September).

All day on 25 September there will be storytelling, singing, language games and other activities for children.

Finally, there's still time for EU secondary schools to register for this year's 'Juvenes Translatores' translation contest. The registration deadline is 20 October and the contest will be held on 24 November. 


\title{
La fiesta de las lenguas en Europa
}

Cultura, educación y juventud - 24/09/2009

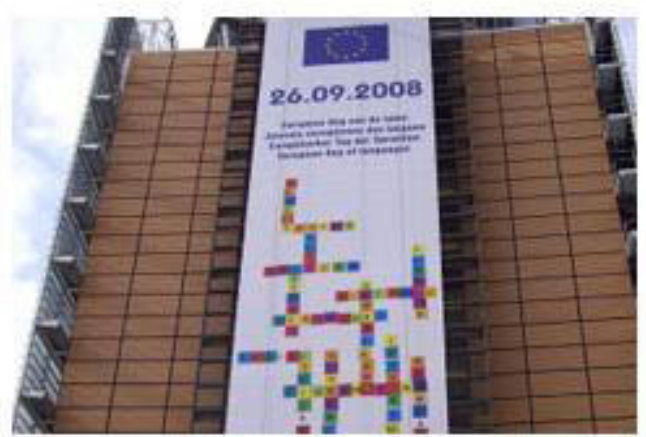

El Día Europeo de las Lenguas celebra la variedad de idiomas hablados en la UE. Infórmese de las actividades organizadas en su país hasta el 26 de septiembre.

Se trata de suscitar el interés por las lenguas y su aprendizaje por niños y adultos. Así que no importan ni su edad ni lo que le lleve a interesarse por las lenguas; seguro que habrá algo que le llame la atención en los más de 300 eventos organizados en toda Europa (al menos 25 en España).

\begin{abstract}
Habrá de todo, desde lo más liviano hasta lo más erudito, desde juegos y concursos hasta veladas literarias y mesas redondas sobre política lingüística.

En Madrid el Instituto Cervantes celebra una jornada de puertas abiertas en la que participarán siete instituciones culturales nacionales (además del Cervantes, el Institut Français, la Nederlandse Taalunie, el Goethe Institut, el British Council, el Instituto Camões y el Nordic Council). En Barcelona, Linguamón organiza junto con la Representación de la Comisión Europea un acto en torno a la relación entre las lenguas y la economía.
\end{abstract}

En Bruselas y Luxemburgo (sedes de la Comisión Europea) habrá una conferencia y un encuentro con la prensa en torno al aprendizaje temprano de lenguas con la asistencia del Comisario de Multilingüismo, Leonard Orban ( 24 y 25 de septiembre).

Para los más pequeños, a lo largo de la jornada del 25 de septiembre

Otro ejemplo es el caso de los artículos que se refieren a actividades comemorativas u otras, donde se intenta, siempre que sea posible, cambiar los ejemplos dados por eventos más cercanos al lector (véase el artículo sobre "La fiesta de las lenguas en Europa" (http://ec.europa.eu/news/culture/090924 es.htm). 